\title{
Software-Defined Approach for Communication in Autonomous Transportation Systems
}

\author{
Piyush Dhawankar ${ }^{1}$, Mohsin Raza $^{1}$, Dr. Hoa Le-Minh ${ }^{1}$ and Dr. Nauman Aslam² \\ ${ }^{1}$ Math, Physics and Electrical Engineering Department, Northumbria University, United Kingdom. \\ ${ }^{2}$ Department of Computer Science and Digital Technologies, Northumbria University, United Kingdom.
}

\begin{abstract}
Autonomous driving technology offers a promising solution to reduce road accidents, traffic congestion and fuel consumption. The management of vehicular networks is challenging as it demands mobility, location awareness, high reliability and low latency of data traffic. In this paper, we propose a novel communication architecture for vehicular network with 5G Mobile Networks and SDN technologies to support multiple core networks for autonomous vehicles and to tackle the potential challenges raised by autonomous driving vehicles. Data requirements are evaluated for vehicular networks with respect to number of lanes and cluster size, to efficiently use frequency and bandwidth. Network latency requirements are analysed, which are mandatory constraints for all applications where real time end-to-end communication is necessary. A test environment is also formulated to evaluate improvement in vehicular network using SDN-based approach over traditional core networks.
\end{abstract}

Keywords: Autonomous driving Vehicles (ADVs); Software Defined Network (SDN); Network Function Virtualization (NFV); Vehicleto-Vehicle (V2V); Vehicle-to-Infrastructure (V2I); Vehicle-to-Everything (V2X); Road-Side-Units (RSUs); On-Board-Units (OBUs); Evolved Packet Core (EPC).

Received on 27 July 2017, accepted on 13 July 2017, published on 14 July 2017

Copyright (C) 2017 Piyush Dhawankar et al., licensed to EAI. This is an open access article distributed under the terms of the Creative Commons Attribution licence (http://creativecommons.org/licenses/by/3.0/), which permits unlimited use, distribution and reproduction in any medium so long as the original work is properly cited.

doi: 10.4108/eai.14-7-2017.152924

\section{Introduction}

The automotive industry has recently shifted from developing advanced vehicles to smart transportation, which focuses on the evolution of new intelligent vehicles for autonomous driving and control capabilities [1]. The autonomous driving vehicles (ADVs) are highly-complex multidisciplinary products, which integrate sensors, automotive control, information processing, artificial intelligence and ultrafast communication capabilities. Governments and society can substantially benefit from autonomous driving, as it will minimise road accidents, help with traffic regulation and optimise usage of fuel and road resources [2]. In order to incorporate autonomous driving, vehicles should be capable of sensing the environment and performing control and path planning without any human intervention [3]. Global automakers and information technology companies, such as General Motors, Volkswagen, Toyota, and Google, expect to have ADVs on the market in 2020 and for $25 \%$ of the vehicles on the road to be ADVs by 2035 [4]. Nevertheless, several challenges still need to be fully addressed for autonomous driving [5, 6], such as:

- To have knowledge of the exact position of the vehicle and to decide how to reach the destination optimally.

- To comprehensively sense the surrounding environment, including other road users and the road infrastructure, to avoid collisions and accidents.

- To detect road signs as well as other static infrastructure details such as lanes, crosswalks, speed bumps, etc.

In existing technologies, sensing systems using a range of cameras, radar, laser range finders and advanced autonomous driving algorithms are employed for this purpose. Nevertheless, current technology is still far from perfect. Furthermore, due to the lack of communication ability among neighbouring vehicles autonomous driving vehicles cannot fully predict the behaviour of neighbouring vehicles. The main 
approach to detect surrounding environments utilises sensor systems, which could be highly affected in different driving conditions (e.g. road/user obstacles, other vehicle behaviours, poor weather conditions). Thanks to the rapid development of wireless communication technologies, vehicular networks are expected to support and boost the development of autonomous driving, and employ Vehicle-to-Vehicle (V2V) and Vehicleto-Infrastructure (V2I) communication techniques, which can be effectively used to detect surrounding conditions [7]. V2V and V2I communication can serve as a second layer of protection in autonomous driving, where every vehicle can periodically broadcast safety-related messages with its current parameters (speed, position, acceleration etc.) to its neighbouring vehicles, which can help other vehicles accurately map their surroundings [8].

The establishment of a reliable communication infrastructure, capable of handling the critical information originating from the vehicular network, needs significant changes in the existing network architecture. Since conventional networks are mostly hierarchical, built with tiers of Ethernet switches arranged in a tree structure, they are only suitable for static networks, thus cannot facilitate the dynamic traffic flow in vehicular networks. The present network architecture, although suitable for client-server computing, continues to pose serious limitations to dynamic computing and storage needs of today's enterprise data centres. Limitations are also posed to carrier environments, which will act as a backbone and service provider for the future ADVs to communicate driving and safety information with the neighbouring vehicles [9].

To facilitate the communications among ADVs and overcome limitations of the traditional network architecture, certain technologies are at hand. Network Function Virtualization (NFV) and Software Defined Networking (SDN) can be termed as possible solutions to handle ever-increasing communication requirements of the next generation ADVs. Network virtualization has greatly increased the number of hosts requiring network connectivity and fundamentally altered assumptions about the physical location of hosts [10]. The benefits of SDN can be realised throughout the network from access to mobile backhaul to the Evolved Packet Core (EPC), thus leveraging SDN's flow-based paradigm, granular policy management, network virtualization and traffic steering capabilities [11].

V2X communications in future cellular/5G (and beyond) mobile network technology are not only relying on evolution of the radio technologies, but new End-to-End (E2E) converged network and cloud infrastructures. This will play a significant role in smart transportation [12]. From the vehicular network perspective, development of $5 \mathrm{G}$ architecture requires provision of high flexibility, low latency load balancing for data routing and high-capacity of nodes, in order to support extremely-dense-and-heterogeneous scenarios (EDHs) where multiple road users are connected by a robust, reliable and dynamic network, allowing for rapid data transmission with sub-millisecond latency requirements [13].
In our proposed architecture, ADVs communicate with RSUs, depending on the local knowledge of their surrounding environment. The SDN controller will create the policies and rules dynamically according to the requirements of ADVs and share with the RSUs, forwarding resources and knowledge to ADVs for their operational requirements. The data from RSUs is sent to data centres through SDN controllers for further processing. To maintain up-to-date network topology of ADVs, the beacon message is broadcasted periodically allowing vehicles to know the neighbours' location. This message also includes information about traffic data, such as route map, position, speed and sensor data, which is again forwarded to the RSUs for processing.

This paper focuses on Vehicle-to-Everything (V2X) communication technologies for future autonomous driving vehicles. The paper presents a novel communication infrastructure for ADVs. The requirements for V2V, V2I communications and vehicular networks is presented, considering safety requirements and critical time margins. In this paper a four-lane road infrastructure is proposed and data requirements for different $\mathrm{V} 2 \mathrm{~V}$ network ranges are evaluated. Furthermore, to evaluate the performance of the SDN controller in comparison to that of a traditional network, a test scenario is created where the communication link from the RSUs to the control server is investigated. The network is being set up for testing both the traditional network and SDN controller (Open-Flow) based network, to compare the performance and reliability of the two networks, and to investigate the suitability of SDN for future network requirements.

The rest of the paper is organised as follows. Section 2 presents the literature review. System Model of vehicular networks, autonomous driving infrastructure, design, arrangement and devices used for the experiment are presented in Section 3. The data communication requirements and critical communication and response times are presented in section 4. Finally, Section 5 concludes the paper and gives future directions.

\section{Literature Review}

The traffic patterns of mobile networks and cloud-based services are dynamic and unpredictable. The present day static and manually configured transport network is not flexible and dynamic enough to support vehicular networks [14]. The mobile and wireless networks have become primary and sole access methods for more and more application services. The mobile operators must support a high volume of traffic, to aid more sophisticated services along with the preferences for the vehicular networks due to its critical nature. The use of more and more cells in Radio Access Network (RAN) provides the mobile subscribers with onramp to wireless networks. Furthermore, the suitability of smaller cells is significantly compromised in vehicular networks due to the need for frequent hand-offs. To address the listed technical difficulties in V2X communications, SDN and NFV are the key technologies [15]. SDN controls the network in a centralised, systematic and programmable manner by decoupling the forwarding function (data plane i.e. user 
equipment) and network controls (control plane i.e. SDN controller/server), improving the efficiency of vehicular networks by fulfilling the requirements of ADVs [16]. With software-based controllers, network operators are much more flexible in programming, modifying, manipulating and configuring protocols in a centralised way, which improves network functionalities in terms of resource allocation and handling immense network loads in vehicular networks. However, NFV aims at realizing network functions on standard high-performance servers/switches and storage devices using a standard virtualisation technology [17]. Network functions are modularised and connected by software interfaces. The network can be sliced by network virtualisation technology and each slice can apply its own network function combination. The dynamic network and resource allocation can be achieved by network management and orchestration module i.e. MANO system [15].

For ADVs, it is important to acquire and communicate information related to position, acceleration, deceleration, speed, steering tilt angle, separation between the vehicles and object tracking. The following objectives are achieved with multiple sensors including Accelerometer, Radar System, Vehicle Dynamics Control (VDC), Differential Global Positioning System and Digital Steering Angle System. Specifications of selected sensors are presented in Table 1. These sensors serve five important aspects of autonomous driving: Localisation, Perception, Planning, Vehicle Control and System Management [18]. Technical details of the selected sensors are provided in Table 1. The sampling rate and the data bits required for communicating the listed sensor values in Table 1 define the data requirements for individual vehicle and can be linearized to larger networks. Furthermore, technical requirements and Key Performance Indicators (KPIs) for autonomous vehicles and V2X use cases are listed below.

- End-to-end latency for automated overtaking (ms): Maximum tolerable elapsed time from the instant, data packet is generated at the source application (ADVs) to the instant it is received by the destination application (Vehicular Network) should be approximately $10 \mathrm{~ms}$ to create the necessary gap in time to avoid a collision with an oncoming vehicle [19].

- Reliability $\left(\mathbf{1 0}^{-\boldsymbol{x}}\right)$ : Maximum tolerable packet loss rate at the application layer, will be $10^{-5}$ within the maximum tolerable latency [19].

- Data rate (Mbit/s): Minimum required data rate for the multiple ADVs applications to function correctly is in a range of 3 to $27 \mathrm{Mbps}$ for exchanging Basic Safety Message (BSM), which contains information on GPS location, speed, direction, and vehicle related information [7].

- Communication range (m): Maximum distance between source and destination(s) of a radio transmission within which the application should achieve the specified reliability. The typical range will be 100 to 300 meters [19].
- Node mobility $(\mathbf{K m} / \mathbf{h})$ : Maximum relative speed under which the specified reliability should be achieved, considering minimum $25 \mathrm{~km} / \mathrm{hr}$ and 120 $\mathrm{km} / \mathrm{hr}$ maximum [20].

- Network density (vehicles $/ \mathrm{km}^{2}$ ): Maximum number of vehicles per unit area under which the specified reliability should be achieved. The saturation point per square kilometre is 2000 vehicles [19].

Table 1. Sensor Data

\begin{tabular}{|c|c|c|c|c|c|}
\hline $\begin{array}{l}\text { Sensor } \\
\text { Type }\end{array}$ & $\begin{array}{l}\text { Referen } \\
\text { ce }\end{array}$ & $\begin{array}{l}\text { Manufa } \\
\text { cturer }\end{array}$ & $\begin{array}{l}\text { Bits/ } \\
\text { Sample }\end{array}$ & $\begin{array}{l}\text { Sample/ } \\
\text { s }\end{array}$ & $\begin{array}{l}\text { Samplin } \\
\text { g Rate } \\
(\mathrm{Hz})\end{array}$ \\
\hline \multirow{2}{*}{$\begin{array}{l}\text { Accelero } \\
\text { meter } \\
{[21][22]}\end{array}$} & $\begin{array}{l}\text { SCA31 } \\
\text { 00-D04 }\end{array}$ & $\begin{array}{l}\text { VTI } \\
\text { Tech }\end{array}$ & 36 & 2000 & 2000 \\
\hline & MM5.10 & Bosch & 36 & 1000 & 2000 \\
\hline $\begin{array}{l}\text { Mid- } \\
\text { Range }\end{array}$ & Front & Bosch & - & - & - \\
\hline $\begin{array}{l}\text { Radar } \\
\text { (MRR) } \\
\text { [23] }\end{array}$ & Rear & Bosch & - & - & - \\
\hline $\begin{array}{l}\text { LiDAR } \\
\text { System } \\
{[24]}\end{array}$ & LUX & IBEO & 14 & 50 & 50 \\
\hline $\begin{array}{l}\text { VDC } \\
{[25]}\end{array}$ & $\begin{array}{l}\text { SMB } \\
225\end{array}$ & Bosch & $\begin{array}{l}16 \\
\text { bits/s }\end{array}$ & 100 & $57 / 180$ \\
\hline $\begin{array}{l}\text { Roll- } \\
\text { over } \\
{[26]}\end{array}$ & $\begin{array}{l}\text { SMB } \\
200\end{array}$ & Bosch & $\begin{array}{l}10 \\
\text { bits/s }\end{array}$ & - & 52 \\
\hline $\begin{array}{l}\text { DGPS } \\
{[27]}\end{array}$ & $\begin{array}{l}\text { DSM13 } \\
2\end{array}$ & Trimble & - & - & $\begin{array}{l}1,2,5 \\
10\end{array}$ \\
\hline $\begin{array}{l}\text { Steering } \\
\text { angle } \\
{[28]}\end{array}$ & 6002 & Bourns & 8 & 100 & 200 \\
\hline
\end{tabular}

For vehicle safety requirements, each vehicle must maintain the safe following distance ( 3 seconds) from the vehicles in front and back. These requirements can significantly change with weather conditions (rain, fog, snow and lightning), traffic and road conditions. Therefore, in an inclement situation, 6 to 9 seconds margin should be maintained [29]. With the safe following distance, knowledge of how fast the vehicle is moving to predict its stopping time and distance is important. For different speeds, the stopping distance and perception time/distance are presented in Table 2. Since the average driver would take one-half to three-quarters of a second to perceive a need to brake, and another three-quarters of a second to move their foot from the gas to the brake pedal. This perception and reaction time in autonomous vehicles is very important and appropriate information must be communicated within due time to take suitable actions. It is also worth noting that the autonomous transportation system must respond within the same duration for good weather conditions, as well as critical. This can be ensured by following recommended separation between vehicles as represented in Table 2. The separation is represented in 
seconds as a general convention, applicable to larger range of speeds and different weather conditions [20].

Table 2. Safe Breaking and Following Distance [20]

\begin{tabular}{lllll}
\hline $\begin{array}{l}\text { Speed } \\
\text { (mph, }\end{array}$ & $\begin{array}{l}\text { Perception } \\
\text { distance } \\
\text { ft./sec) }\end{array}$ & $\begin{array}{l}\text { Overall } \\
\text { (ft.) }\end{array}$ & $\begin{array}{l}\text { Stopping } \\
\text { Distance } \\
\text { (ft.) }\end{array}$ & $\begin{array}{l}\text { Safe following } \\
\text { distance }\end{array}$ \\
\cline { 4 - 5 } & & $\begin{array}{l}\text { Good } \\
\text { (ft.) }\end{array}$ & $\begin{array}{l}\text { Marginal } \\
\text { (ft.) }\end{array}$ \\
$(25,37)$ & 37 & 74.16 & 111 & 222 \\
$(35,52)$ & 52 & 103.83 & 166 & 312 \\
$(45,66)$ & 66 & 133.497 & 198 & 396 \\
$(55,81)$ & 81 & 163.163 & 243 & 486 \\
$(65,96)$ & 96 & 192.829 & 288 & 576 \\
$(75,111)$ & 111 & 222.495 & 333 & 666 \\
\hline
\end{tabular}

Time separation between the vehicles ( 3 seconds /Good weather) and ( 6 seconds / Critical weather)

\section{System Model and Design of Experiment}

\subsection{System Model}

For V2V communication, Time Division Multiple Access (TDMA) is used, based channel access, allowing vehicles to communicate in dedicated slots to reduce interference. The interference between the two clusters is mitigated by using clustered structure with $n$ Frequency sets. In Figure 1, the four-lane road infrastructure for ADVs is considered and as an example scenario, three clusters are represented. The ADVs in a cluster exchange information among each other in regular intervals, whereas the inter-cluster communication is executed by RSUs. Each cluster uses a dedicated frequency $(f 1, f 2, f 3)$ to mitigate inter-cluster interference. The cluster size can extend to multiple-hops where each additional hop adds more vehicles in front and back of the cluster, represented by figure 1 .

The proposed architecture for V2X communication is shown in Figure 2, whereas the low-level cellular infrastructure (as represented in Figure 1) is integrated with the higher hierarchy of the network model. In the proposed architecture, ADVs act as dynamic nodes, installed with wireless OnBoard Units (OBUs) for communicating with other ADVs and infrastructure. The OBUs communicate in vehicular network with the help of Road Side Units (RSUs). The ADVs are equipped with multiple sensors such as pre-crash collision sensors; adaptive cruise control sensors; blind spot detection sensors and rear-crash collision sensor etc. These sensors provide vehicles with complete perspective of road infrastructure, and any objects in its proximity, to facilitate smooth and safe driving.

The proposed architecture is implemented using SDN and cellular structure to facilitate frequency reuse, and accommodate large number of ADVs and related applications. The cellular base station (eNB) is under the control of SDN controller and facilitates local vehicular network. Multiple RSUs are connected to the RSU controller and are responsible for forwarding data, storing local road information and performing emergency services.

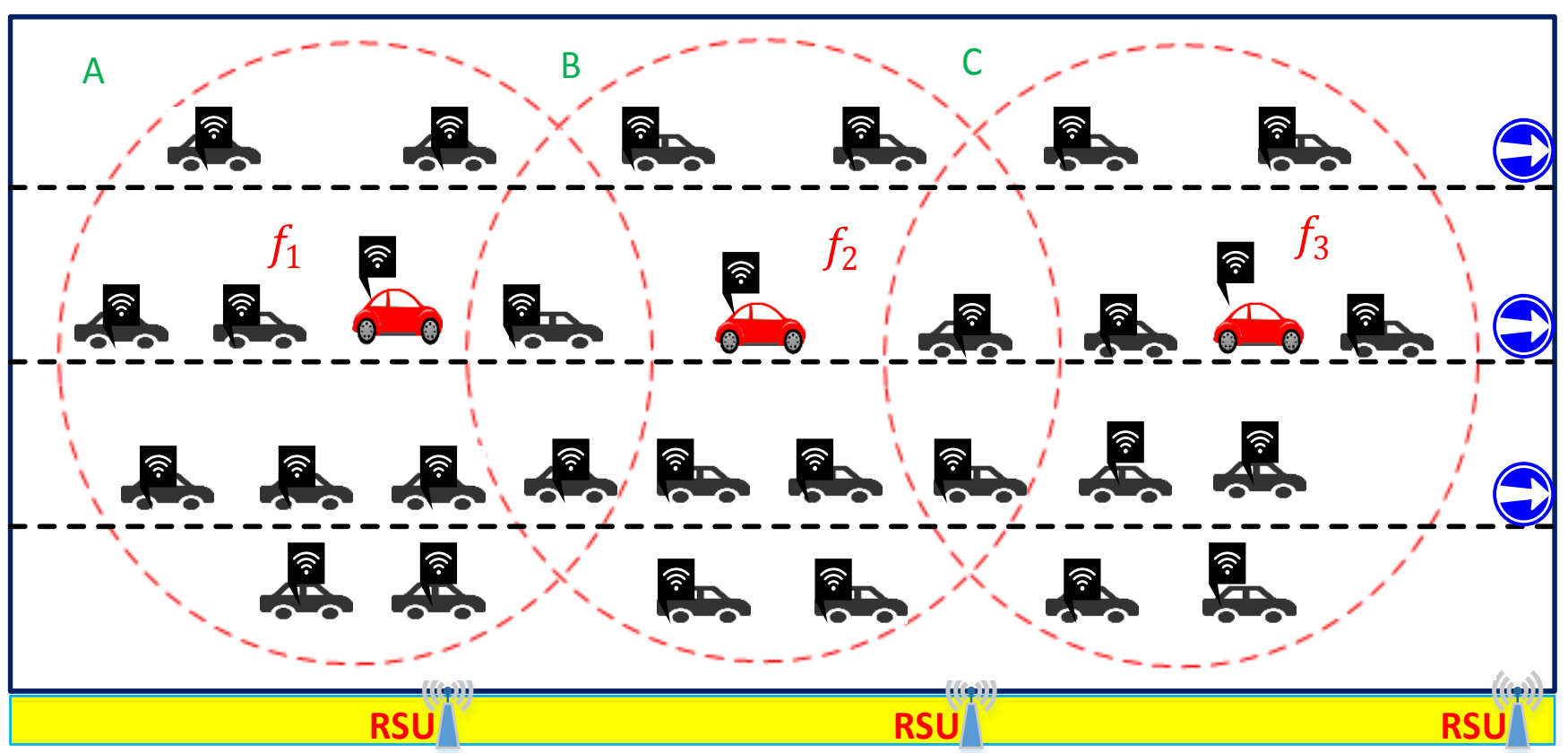

Figure 1. Four Lane Road Infrastructure for ADVs 

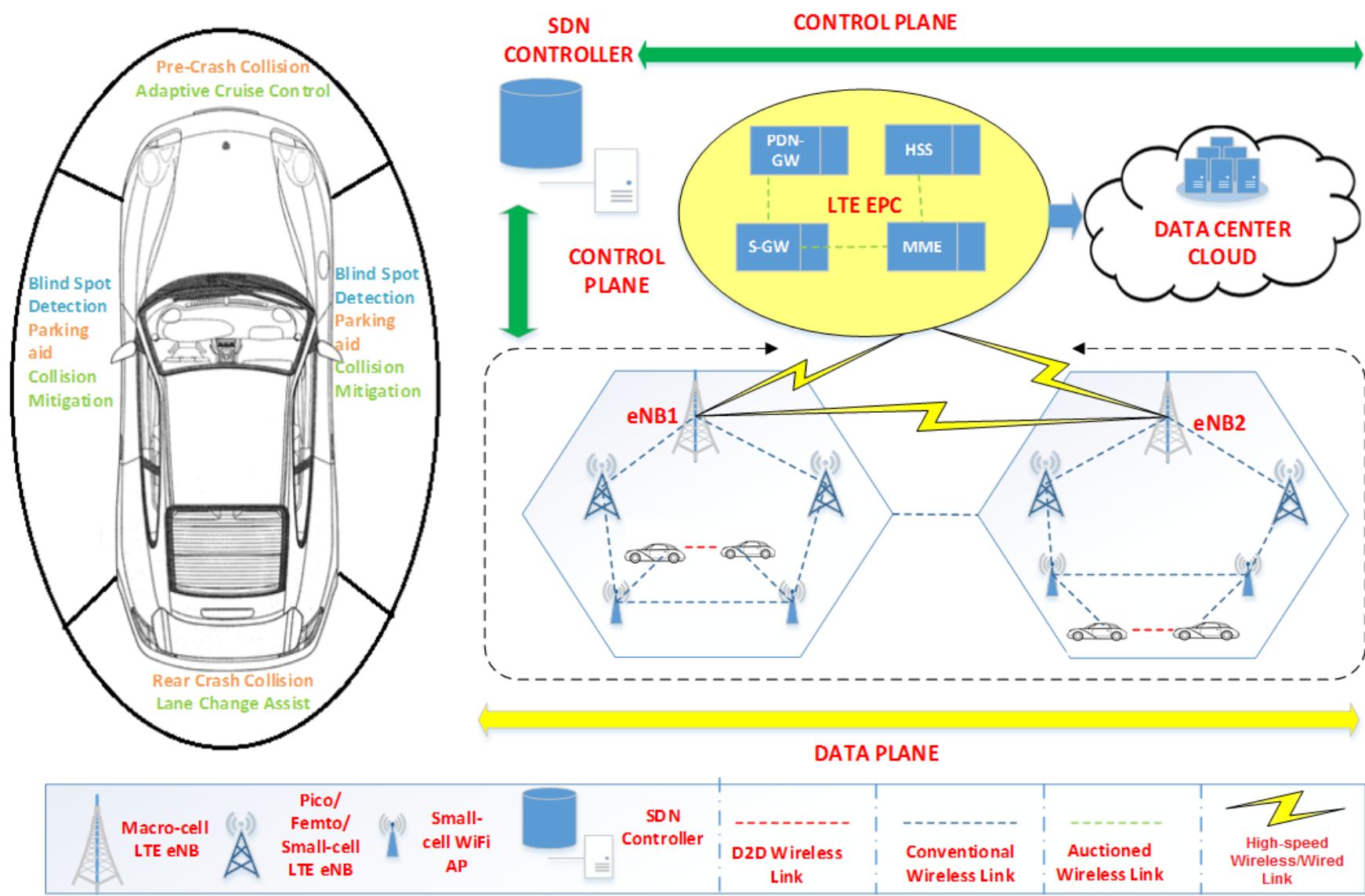

Figure 2. Proposed Architecture for ADVs

The RSUs are responsible of accumulating sensory data from ADVs. With the increase in cluster-size, or while facilitating multiple clusters, RSUs accumulate data from the affiliated ADVs. However, due to the large amount of data received from ADVs, the links from RSUs to RSU controller can saturate. Evaluation of management capabilities of the high-speed data link from RSUs to RSU controller is important. Further to this, it is significantly important that resources are efficiently managed and are equally divided in the multiple RSUs for effective operation.

\subsection{Design of Experiment}

Seamless communication between vehicles and infrastructure is a requirement of vehicular networks. The communication between the ADVs and RSUs is wireless, with RSUs communicating with the core network via an SDN controller to provide network services. The traditional core network is an insufficient competent to support vehicular networks, as the requirements of vehicular networks are always dynamic. The processing time for the resource allocation is very stringent, requiring remodeling of the traditional network to support such demand for vehicular network. In this scenario, the efficiency and throughput of traditional core network and SDN-based network is evaluated. The test scenario is being created with Cisco devices and HP SDN compatible devices, where hosts act as RSUs and the server acts as core network with TCP based traffic flow. Figure 3 shows the network setup to conduct the throughput tests from hosts (RSUs) to the server (Core Network). Two topologies are being created for the tests via Cisco topology and SDN topology. The devices with the same configuration are being used, with parameters maintained the same for network testing tools, to investigate the difference between the throughputs from both test scenarios. The difference between Cisco and SDN topology is the presence of the SDN controller for SDN topology management, which is not the case in Cisco. The SDN controller has a global view of all the devices, allowing it to manage networks more appropriately. It is very convenient for network operators to decide how the traffic is routed and application of certain policies to route network traffic dynamically according to the needs of specific applications is possible.

The equipment used for the test scenarios is presented in Table 3. For the network formation, two Cisco layer 3 switches are used as a distribution and access layer for Cisco topology, while two HP switches with SDN (OpenFlow) compatibility are used for SDN topology. The host and server machines are running Windows 7 Enterprise, with AMD processor and 8 GB of RAM. The SDN controller is running on Ubuntu 16.04 with VMware virtualisation desktop and used only for SDN topology. To measure the throughput and performance of network by 
varying the parameters such as payload, protocol, etc. is managed by JPerf graphical interface open source software. It accounts parameters such as bandwidth, delay, and jitter amongst others.

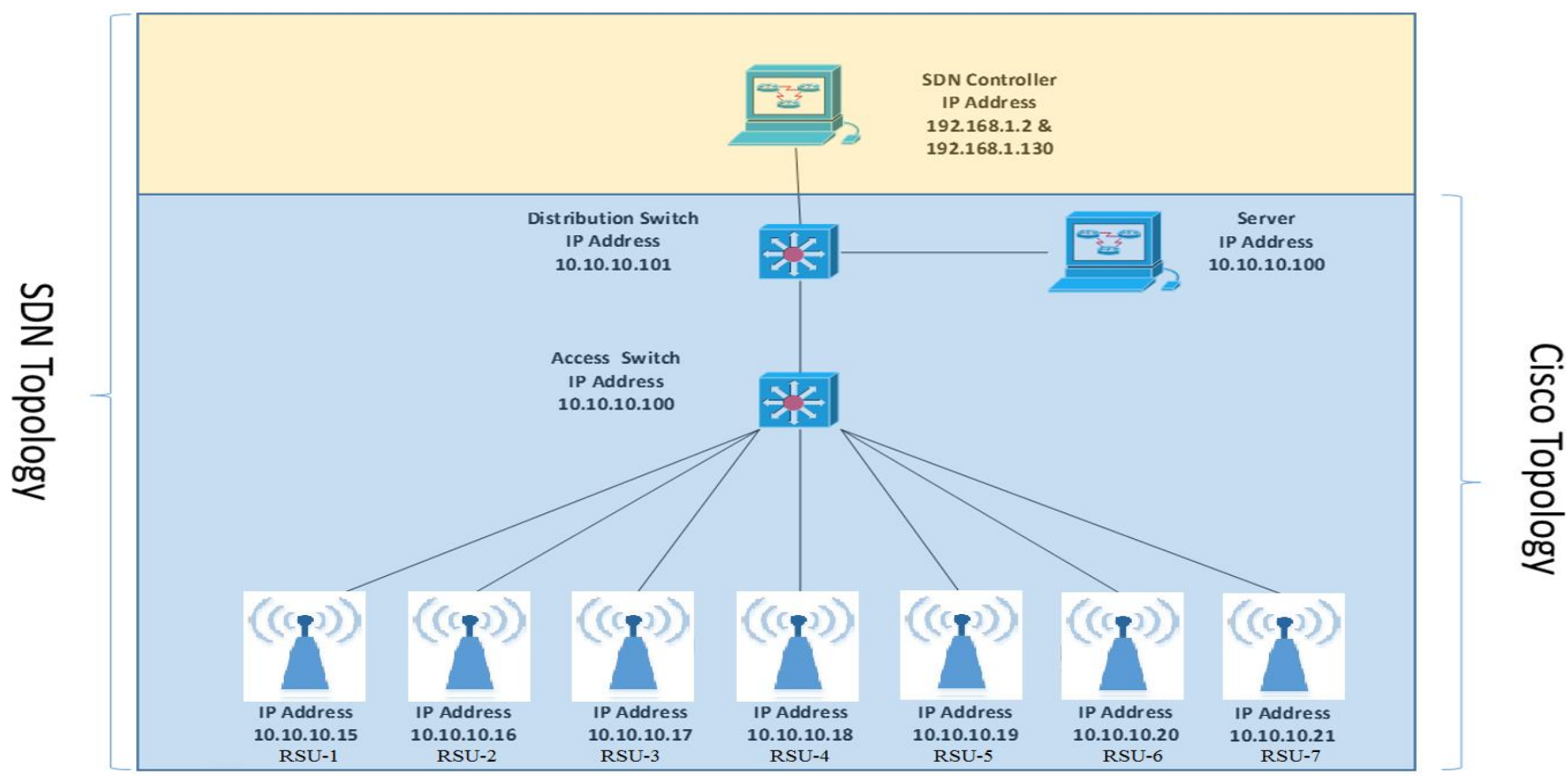

Figure 3. Network Diagram for Throughput Test

Table 3. List of equipment used for setup [30, 31]

\begin{tabular}{lll}
\hline Hardware & Cisco Catalyst & HP Procurve \\
\hline Distribution Layer Switch & WS-C3550-12G & HPE 3800-24G-2SPF+ Switch (J9575A) \\
Access Layer Switch & WS-C3550-12T & HPE 3800-24G-2SPF+ Switch (J9575A) \\
Hosts/Server & Windows 7 Enterprise Service Pack & Windows 7 Enterprise Service Pack 1 64-bit \\
& 164- bit with AMD A4-5300 APU & with AMD A4-5300 APU with Radeon TM HD \\
& with Radeon TM HD Graphics 3.40 & Graphics 3.40 GHz with 8 GB RAM. \\
& GHz with 8 GB RAM. & Ubuntu 16.04, 64 bit with AMD A4-5300 APU \\
NDN Controller & N.A. & with Radeon TM HD Graphics 3.40 GHz with 8 \\
& & GB RAM. \\
Network Testing Tool & Jperf version 2.0.2 & \\
\hline
\end{tabular}

\section{Results and Discussion}

The proposed low level cellular infrastructure in Figure 1 is a four-lane road scenario with 3 clusters labelled as A, B and $\mathrm{C}$. Each cluster has number of vehicles which can be handled by the local vehicular network. The data requirement for each cluster is dependent on the number of ADVs and number of lanes (road). The data requirements vary with the lane traffic and different cluster sizes (in case the cluster represented in figure 1 is extended to multi-hops of communication area).

For the communication in each cluster, data requirements can change drastically for different number of lanes and cluster sizes. Figure 4 presents upper bound on data requirements in vehicular communication networks for three different cluster-sizes and different number of lanes. The required data rate is evaluated using the sampling rate and quantisation levels used for each sensor, mandatory for giving sufficient information for autonomous driving. The susceptibility of packet loss in wireless channel is also considered for the evaluation of the maximum data link requirements under given conditions. A TDMA based slotted channel access is implied where control information necessary to handle the network traffic is also included in evaluation. Since the sensory data necessary to predict vehicle trajectory and movement is relatively low ( 1 kbyte), it can easily be handled with traditional wireless networks. To keep the network updated, the sensor data from the vehicles is communicated every 100 milliseconds. The increase in a cluster size allows more accommodation of ADVs, resulting in better network management. However, the data requirements change notably. 


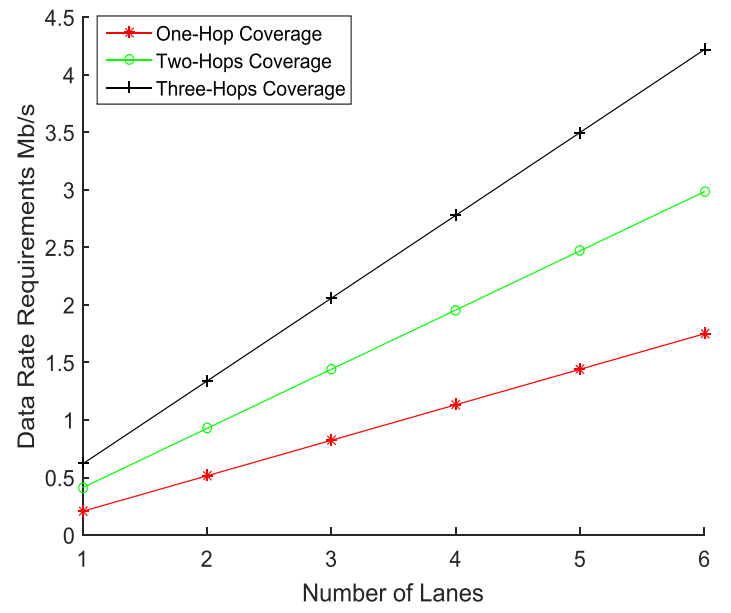

Figure 4. Maximum Data Requirements for Different Number of lanes

Table 4. Cisco wired TCP

\begin{tabular}{cccccccc}
\hline Run & Host & Host & Host & Host & Host & Host & Host \\
s & 1 & 2 & 3 & 4 & 5 & 6 & 7 \\
\hline $\begin{array}{c}\text { Run } \\
1\end{array}$ & 738 & 924 & 927 & 882 & 916 & 926 & 933 \\
$\begin{array}{c}\text { Run } \\
2\end{array}$ & 720 & 917 & 925 & 926 & 913 & 896 & 940 \\
$\begin{array}{c}\text { Run } \\
3\end{array}$ & 703 & 913 & 921 & 920 & 922 & 897 & 929 \\
$\begin{array}{c}\text { Run } \\
4\end{array}$ & 724 & 899 & 928 & 946 & 884 & 920 & 932 \\
$\begin{array}{c}\text { Run } \\
5\end{array}$ & 704 & 907 & 927 & 924 & 883 & 897 & 937 \\
$\begin{array}{c}\text { Run } \\
6\end{array}$ & 735 & 911 & 940 & 866 & 902 & 929 & 936 \\
$\begin{array}{c}\text { Run } \\
7\end{array}$ & 697 & 920 & 932 & 917 & 903 & 911 & 929 \\
$\begin{array}{c}\text { Run } \\
8\end{array}$ & 697 & 922 & 932 & 912 & 914 & 928 & 945 \\
$\begin{array}{c}\text { Run } \\
9\end{array}$ & 711 & 919 & 927 & 929 & 924 & 932 & 936 \\
$\begin{array}{c}\text { Run } \\
10\end{array}$ & 694 & 922 & 926 & 897 & 910 & 927 & 940 \\
\hline $\begin{array}{c}\text { Aver } \\
\text { age }\end{array}$ & 713. & 915. & 928. & 911. & 907.1 & 916.3 & 935. \\
\hline
\end{tabular}

In the presented scenario, the test mainly focuses on investigating the throughput against the advertised data rates by using standard protocols. In the presented network scenario, the host sends the continuous stream of data to the server for a fixed period of 10 seconds and the output is recorded in Mbps as throughput data. Since the link is not saturated by only a single stream of data, multiple streams of data are sent simultaneously to the server by multiple hosts. To eliminate outliers, ten runs are recorded and averaged throughput is used. To compare the performance of SDN with the traditional networks, uniform circumstances are ensured for both the cases. Both network scenarios (SDN based and conventional) are tested by increasing the number of parallel streams where the obtained results for conventional and SDN based networks are presented in Table 4 and Table 5 respectively.

Table 5. SDN wired TCP

\begin{tabular}{cccccccc}
\hline Runs & Host & Host & Host & Host & Host & Host & Host \\
& 1 & 2 & 3 & 4 & 5 & 6 & 7 \\
\hline $\begin{array}{c}\text { Run } \\
1\end{array}$ & 877 & 920 & 929 & 957 & 943 & 956 & 957 \\
$\begin{array}{c}\text { Run } \\
2\end{array}$ & 878 & 937 & 948 & 965 & 936 & 942 & 959 \\
$\begin{array}{c}\text { Run } \\
3\end{array}$ & 882 & 936 & 948 & 940 & 946 & 950 & 952 \\
$\begin{array}{c}\text { Run } \\
4\end{array}$ & 872 & 923 & 951 & 945 & 937 & 958 & 959 \\
$\begin{array}{c}\text { Run } \\
5\end{array}$ & 900 & 936 & 932 & 958 & 949 & 957 & 966 \\
$\begin{array}{c}\text { Run } \\
6\end{array}$ & 861 & 940 & 928 & 952 & 951 & 952 & 956 \\
$\begin{array}{c}\text { Run } \\
7\end{array}$ & 867 & 930 & 940 & 955 & 959 & 964 & 958 \\
$\begin{array}{c}\text { Run } \\
8\end{array}$ & 887 & 940 & 945 & 948 & 951 & 948 & 955 \\
$\begin{array}{c}\text { Run } \\
9\end{array}$ & 878 & 941 & 931 & 947 & 949 & 961 & 953 \\
$\begin{array}{c}\text { Run } \\
10\end{array}$ & 862 & 936 & 951 & 949 & 944 & 955 & 962 \\
\hline $\begin{array}{c}\text { Aver } \\
\text { age }\end{array}$ & 867. & 933. & 940. & 951. & 946.5 & 954.3 & 957. \\
\hline & 9 & 3 & 6 & & & 7 \\
\hline
\end{tabular}

The data requirements plotted in Figure 4, indicate the throughput needs of a single cluster with increase in number of lanes and cluster size. Each cluster is affiliated with a particular RSU, while multiple clusters can be associated with single RSU. The data accumulated at the RSU needs to be transmitted to the RSU controller and data center, hence high-speed links are desirable in the core network, with the capability of handling multiple RSUs over a single link. To investigate the throughput in the core network and the effect of increasing host units, the network topology is presented in Figure 3. As per the throughput requirements presented in Figure 4, and network topology presented in Figure 3, maximum load capabilities of SDN controller are evaluated. The core network performance is evaluated with up to seven RSUs where each RSU can facilitate up to twenty-five, 3-hop clusters.

The results show that one host SDN (TCP) performs better than CISCO (TCP). It is also observed that the throughput in case of SDN based network remains far more consistent. The throughput for both state of the art Cisco network and the HP SDN based network is presented in Figure 5, where the performance of the of SDN network remains continuously better than that of the Cisco network. It can be seen that the average throughput achieved by the SDN topology is better than the Cisco topology, as with two RSUs the Cisco (TCP) only manages to achieve 915.7 Mbps while SDN (TCP) achieves 933.9 Mbps, offering 2\% improvement over the traditional network. As the more RSUs are used, the improvement in data transmission and 
efficiency in bandwidth improves up to $4 \%$. Since the $1 \mathrm{Gbps}$ link is used for the above stated cases, the link is already saturated. However, as the number of RSUs are increased, the Cisco and SDN based networks must manage the bandwidth between multiple RSUs. The obtained results suggest that SDN effectively manages the increasing number of RSUs compared to traditional Cisco network. The overall efficiency in terms of throughput enhancement in case of SDN based network is presented in Figure 6.

The evaluated results suggest that SDN based network offers improved throughput and ensures bandwidth consistency in the network, allowing relatively lesser fluctuations in the system bandwidth with a change in circumstances. In case of vehicular networks, SDN would offer better resource allocation by improving utilization of available bandwidth. To conclude with the test on TCP with 1 Gbps link, the TCP (SDN) performs better than TCP (CISCO).

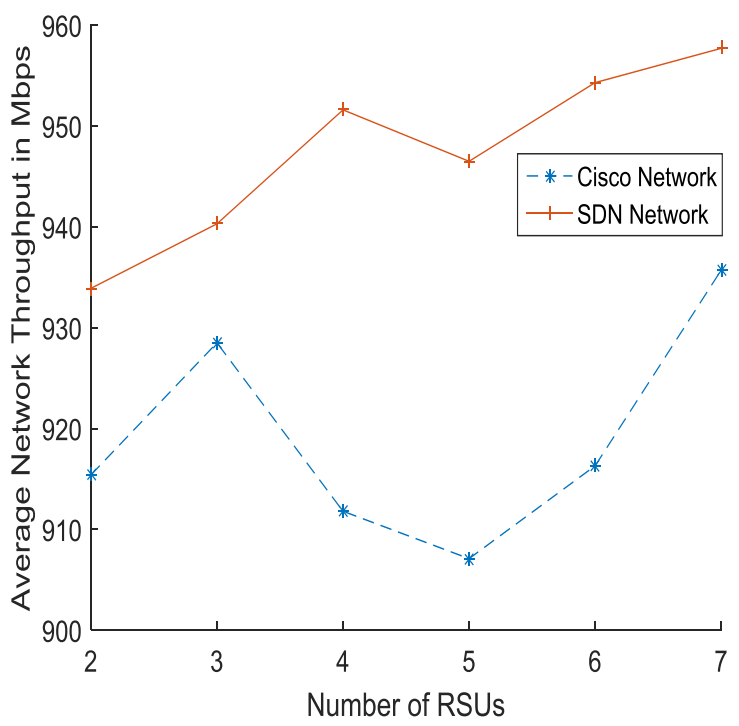

Figure 5. Average throughput of network

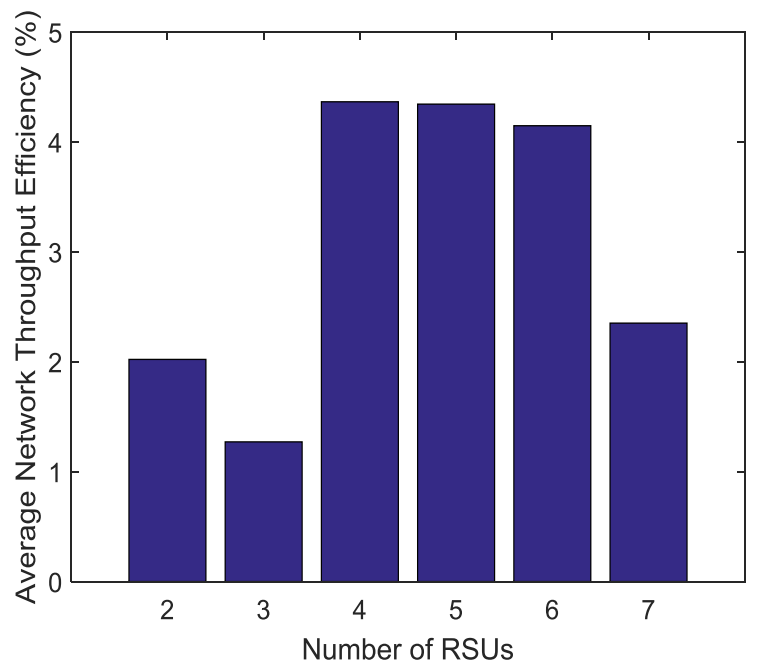

Figure 6. Efficiency of the network

\section{Conclusion and Future Directives}

Autonomous driving technology can serve suitably for future vehicles. However, it is important that the autonomous driving system is extended to network level instead of a stand-alone solution, in order to access the full benefits of the communication technology and to implement a secondary layer of safety. In this paper, a $5 \mathrm{G}$ and SDN based vehicular network was investigated for establishing a reliable link between the vehicles and the intelligent traffic control infrastructure. The use of SDN offers improvements over the conventional networks and reduces bandwidth fluctuation.

The paper lays foundations for ADVs and network architecture, however, the data requirements for these networks need to further investigate the SDN based network attributes. In the vehicular networks, SDN can offer optimised resource management. However, feasibility of Base Station (BS) and RSUs to cooperate with SDN controller for network knowledge to optimise the resource utilisation, service hosting and migration need to be validated. Furthermore, fallback/backup mechanism in case of unreliable connections or failure between RSUs and ADVs, when SDN controller is maintaining the services, must be carefully addressed. In conclusion, it is important to simulate proposed architecture, model, protocols and services using simulator platforms such as Mininet, NS-3, Matlab and Vissim.

\section{References}

[1] K. Jo, J. Kim, D. Kim, C. Jang, and M. Sunwoo, "Development of Autonomous Car \& 2014;Part II: A Case Study on the Implementation of an Autonomous Driving System Based on Distributed Architecture," IEEE Transactions on Industrial Electronics, vol. 62, pp. 5119-5132, 2015.

[2] J. M. Anderson, K. Nidhi, K. D. Stanley, P. Sorensen, C. Samaras, and O. A. Oluwatola, Autonomous Vehicle Technology: A Guide for Policymakers: Rand Corporation, 2014.

[3] C. Urmson, J. Anhalt, D. Bagnell, C. Baker, R. Bittner, M. Clark, et al., "Autonomous Driving in Urban Environments: Boss and the Urban Challenge," Journal of Field Robotics, vol. 25, pp. 425-466, 2008.

[4] J. Bierstedt, A. Gooze, C. Gray, J. Peterman, L. Raykin, and J. Walters, "Effects of next-generation vehicles on travel demand and highway capacity," FP Think Working Group, pp. 10-11, 2014.

[5] J. Choi, J. Lee, D. Kim, G. Soprani, P. Cerri, A. Broggi, et al., "Environment-Detection-and-Mapping Algorithm for Autonomous Driving in Rural or OffRoad Environment," IEEE Transactions on Intelligent Transportation Systems, vol. 13, pp. 974-982, 2012.

[6] R. Yu, J. Ding, X. Huang, M. T. Zhou, S. Gjessing, and Y. Zhang, "Optimal Resource Sharing in 5G-Enabled Vehicular Networks: A Matrix Game Approach," IEEE Transactions on Vehicular Technology, vol. 65, pp. 7844-7856, 2016.

[7] L. Frenzel. (2016, 09/01). Major Wireless Development Driving 2017 Agendas. Available: 
http://electronicdesign.com/wireless/major-wirelessdevelopments-driving-2017-agendas

[8] K. Sasaki, N. Suzuki, S. Makido, and A. Nakao, "Vehicle Control System Coordinated between Cloud and Mobile Edge Computing," in Society of Instrument and Control Engineers of Japan (SICE), 2016 55th Annual Conference of the, 2016, pp. 1122-1127.

[9] Open Networking Foundation (ONF), "SoftwareDefines Networking: The New Norm for Networks", White Paper, Apr., 2013.

[10] Open Networking Foundation (ONF), "SDN in the Campus Environment”, White Paper, Sep., 2013.

[11] Open Networking Foundation (ONF), "OpenFlowEnabled Mobile and Wireless Networks", White Paper, Sep., 2013.

[12] R. Vilalta, A. Mayoral, R. Casellas, R. Martínez, and R. Muñoz, "Experimental Demonstration of Distributed Multi-Tenant Cloud/Fog and Heterogeneous SDN/NFV Orchestration for 5G Services," in Networks and Communications (EuCNC), 2016 European Conference on, 2016, pp. 52-56.

[13] 5GPP. (2015, 09/01). The 5G Infrastructure Public Private Partnership: the Next Generation of Communication Networks and Services (White paper). Available: https://5g-ppp.eu/wpcontent/uploads/2015/02/5G-Vision-Brochure-v1.pdf

[14] Open Networking Foundation (ONF), "OpenFlowenabled Transport SDN", White Paper, May, 2014.

[15] Y. Fengyi, W. Haining, M. Chengli, Z. Jianmin, and W. Min, "A Flexible Three Clouds 5G Mobile Network Architecture Based on NFV \& SDN," China Communications, vol. 12, pp. 121-131, 2015.

[16] N. B. Truong, G. M. Lee, and Y. Ghamri-Doudane, "Software Defined Networking-Based Vehicular Adhoc Network with Fog Computing," in Integrated Network Management (IM), 2015 IFIP/IEEE International Symposium on, 2015, pp. 1202-1207.

[17] M. Chiosi. (2012, 09/01). Network Functions Virtualization: An Introduction, Benefits, Enablers, Challenges \& Call for Action Available: http://portal.etsi.org/NFV/NFV White Paper.pdf

[18] R. Coppola and M. Morisio, "Connected Car: Technologies, Issues, Future Trends," ACM Comput. Surv., vol. 49, pp. 1-36, 2016.

[19] E. C. 5GPP. (2015, 10/01/2017). 5G Automotive Vision. Available: https://5g-ppp.eu/wpcontent/uploads/2014/02/5G-PPP-White-Paper-onAutomotive-Vertical-Sectors.pdf

[20] L. James. (2008, 15/02). Maintain a Safe Following Distance (The 3 Second Rule). Available: http://www.smartmotorist.com/traffic-and-safetyguideline/maintain-a-safe-following-distance-the-3second-rule.html

[21] VTI Technologies, "SCA3100-D04 3-Axis High Performance Accelerometer with Digital SPI Interface Datasheet," ed., 2015.

[22] Bosch, "Acceleration Sensor MM5.10 Datasheet," ed., 2016.

[23] Bosch, "Mid-Range Radar Sensor (MRR) for Front and Rear Application Datasheet," ed., 2015.

[24] IBEO, "LUX Lidar Sensor Datasheet," ed., 2010.

[25] Bosch, "Low-g accelerometer for vehicle dynamics control SMB225 Datasheet," ed., 2013.

[26] Bosch, "Low-g Accelerometer for Rollover Applications SMB200 Datasheet," ed., 2013.
[30] Cisco, "Cisco Catalyst 3550 Series Intelligent Ethernet Switches Datasheet," ed., 2017

[31] HP, "HPE 3800 Switch Series Datasheet," ed., 2016

Trimble, "DSM132 DGPS Receiver Datasheet," ed., 2005.

Bourns, "Non-Contacting Steering Angle Sensor Type 6002 Datasheet," ed, 2013 